\title{
CONCERNING A BOUND PROBLEM IN KNOT THEORY
}

\author{
BY \\ L. B. TREYBIG
}

\begin{abstract}
In a recent paper Treybig shows that if two knot functions $f, g$ determine equivalent knots, then $f, g$ are the ends of a simple sequence $x$ of knot functions. In an effort to bound the length of $x$ in terms of $f$ and $g(1)$ a bound is found for the moves necessary in moving one polyhedral disk onto another in the interior of a tetrahedron and (2) it is shown that two polygonal knots $K, L$ in regular position can "essentially" be embedded as part of the 1-skeleton of a triangulation $T$ of a tetrahedron, where (1) all 3 cells which are unions of elements of $T$ can be shelled and (2) the number of elements in $T$ is determined by $K, L$. A number of "counting" lemmas are proved.
\end{abstract}

Introduction. The work in this paper is a continuation of research carried on in [3], [7], [9], [12], [13], [14], [15]. For a more detailed explanation of the ideas behind the problem attacked here see [15].

The theorems proved here are results of attempts by the author to settle a conjecture of the following type: Suppose a piece of rope in the shape of a simple closed curve is moved in space so as to form at different times two knots whose projections have no more than $n$ crossings. Then, the rope can be moved in small steps from the first position to the second one so that its projection never has more than $2 n$ crossings.

This type of problem can be considered as a special case of the more general problem: "Suppose $K_{1}$ and $K_{2}$ are finite complexes whose elements are rectilinear and lie in the interior of an $n$-simplex $S$, and $f: S \rightarrow S$ is an onto piecewise linear homeomorphism such that (1) $f(x)=x$ if $x \in \mathrm{Bd}(S)$, and (2) $f\left(\left|K_{1}\right|\right)=\left|K_{2}\right|$. Then, determine a bound $B$ stated in advance in terms of card $\left(K_{1} \cup K_{2}\right)$ such that there is a triangulation $T$ of $S$ and a simplicial isotopy $h: S \times[0,1] \rightarrow S$ such that (1) $h_{t}(x)=x$ if $t=0$ or $x \in \mathrm{Bd}(S),(2) h_{1}\left(\left|K_{1}\right|\right)=\left|K_{2}\right|$, (3) $h_{t}$ is affine on each simplex in $T$ for each $t$ in $[0,1]$, and (4) card $(T) \leqq B$." A major difficulty here is that $f$, when thought of as the final stage of an isotopy, may take $\left|K_{1}\right|$ and then "twist" things quite a bit more than necessary, and then finally place $\left|K_{1}\right|$ on $\left|K_{2}\right|$.

One type of problem in which a bound has been successfully located is the word problem for certain types of finitely presented groups [4], [5], [6]. In a forthcoming paper the author uses results analogous to those of Greendlinger [6] to show that, if $K_{1}$ and $K_{2}$ are polygonal knots in regular position in $E^{3}$ which are equivalent

Received by the editors February 10, 1970.

AMS 1969 subject classifications. Primary 1188; Secondary 1184.

Key words and phrases. Polyhedron, polygonal knot, piecewise linear homeomorphism, simplicial isotopy, free cell, shelling order. 
under an orientation preserving homeomorphism of $E^{3}$ onto $E^{3}$, then there is a finitely presented group $|X: R|$ such that (1) the word problem is solvable in $|X: R|,(2) K_{1}$ and $K_{2}$ are represented as words $w_{1}$ and $w_{2}$, respectively, (3) $w_{1}$ is a reduction of a word $\left(\prod_{p=1}^{n} g_{p}^{-1} r_{p} g_{p}\right) w_{2}$, where if the reduction is carried out in certain "small" steps, then $K_{2}$ is moved to $K_{1}$ in certain "small" steps. The group is constructed using (1) results in this paper and in [15] to obtain a special triangulated 3-cell and (2) certain methods analogous to those of Neuwirth [8] to relate the algebra and the geometry.

$\$ 3$ contains several lemmas which count the number of simplexes in certain triangulations. In $\$ 4$ a special case of the above general problem is handled; namely the one where the $\left|K_{i}\right|$ are polyhedral disks embedded in the interior of a tetrahedron. Also in $\$ 4$ a theorem is proved which "essentially" shows how two polygonal knots can be embedded as a union of 1 -simplexes of a triangulation $T$ of a tetrahedron $M$, where (1) the number of 3-simplexes in $T$ is stated in terms of the given knots and (2) every 3-cell which is the union of two or more 3-simplexes of $T$ has two such simplexes that are free.

2. Definitions. Most of the terms used in this paper are defined in one of [1], [2], [10].

All of the spaces considered are subsets of Euclidean 3-space $E^{3}$, and all triangulations of such spaces will be locally finite and have closed simplexes which are rectilinear (i.e., are points, straight line intervals, triangular disks or two cells, or solid tetrahedra).

A subset $S$ of $E^{3}$ will be called a polyhedron, or be said to be polyhedral, if it has a rectilinear triangulation. A mapping $f: S \rightarrow T$ between polyhedra will be said to be piecewise linear (p.l.) if there is a rectilinear triangulation $W$ of $S$ such that $f$ is affine on each simplex of $W$. If $f, f^{\prime}: S \rightarrow T$ are p.l. homeomorphisms, then we say $K: S \times[0,1] \rightarrow T$ is a p.l. isotopy between $f$ and $f^{\prime}$ if $(0) K$ is continuous, (1) $K(s, 0)=f(s)$ and $K(s, 1)=f^{\prime}(s)$ for $s \in S$, and (2) for each $t \in[0,1] K_{t}$ is a p.l. homeomorphism. Furthermore, $K$ will be said to be a simplicial isotopy if some fixed triangulation $W$ of $S$ can be found so that $K_{t}$ is affine on each $w \in W$ for all $t \in[0,1]$.

If $T$ is a triangulation of a polyhedron $S$, we let $n\left(T_{p}\right)=\operatorname{card}\left(T_{p}\right)$, where $T_{p}$ denotes the collection of $p$-simplexes of $T$. Also, for any $T^{\prime} \subset T$, let $\left|T^{\prime}\right|=\bigcup T^{\prime}$. Given $M \subset S$ let st $(M, T)$ denote the collection of all simplexes of $T$ which contain $M$.

If $M$ is an $n$-manifold with boundary then $\mathrm{Bd}(M)$ will denote the set of all points of $M$ which do not have a neighborhood in $M$ homeomorphic to $E^{n}$. If $M$ is an $n$-cell, then an $n$-cell $N \subset M$ will be said to be free in $M$ provided $M=N$ or $N \cap \mathrm{Bd}(M)$ is an $n-1$ cell. If $P \in E^{n}$ and $M \subset E^{n}$ then the cone over $M$ from $P$ will denote the union of all intervals $P m$, where $m \in M$. Denote this cone by $P M$. Unless otherwise stated, by interval we mean straight line interval. 
A polygonal knot $K$ is a simple closed curve in $E^{3}$ which is the union of a finite collection $\left\{a_{1} a_{2}, a_{2} a_{3}, \ldots, a_{n} a_{1}\right\}$ of intervals. The knot $K$ will be said to be in regular position provided the intervals can be selected so that the $x y$ projection $\pi a_{i}$ of each $a_{i}$ is not the $x y$ projection of any other point of $K$, and no point is the $x y$ projection of more than two points of $K$. The points that are the projection of two such points will be called the double points or crossing points of the projection.

3. Preliminary lemmas. In this section we prove several lemmas which are necessary for the main theorems in $\S 4$.

Lemma 0. Suppose $D$ is a polyhedral 2-cell with rectilinear triangulation $T$. Then, if $n\left(T_{2}\right)>1$, there exist two elements $t_{1}, t_{2}$ of $T_{2}$ such that $(1) t_{i}(i=1,2)$ is free in $D$, and $(2)$ if $t_{i}(i=1,2)$ has a 0 -simplex $v_{i}$ in Int $(D)$, then $\left|s t\left(v_{i}, T\right)\right|$ is free in $D$, and $v_{1} \neq v_{2}$ unless $T_{0} \cap \operatorname{Int}(D)=\left\{v_{1}\right\}$.

Proof. By Lemma 2 of [10] there exist two elements $s, s^{\prime}$ of $T_{2}$ that are free in $D$. Suppose that $\mid$ st $(v, T) \mid$ is not free in $D$ for some vertex $v$ of $s$ in Int $(D)$. Each component $C$ of $D-\mid$ st $(v, T) \mid$ has the property that $\bar{C}$ is a 2-cell and $\bar{C} \cap|\mathrm{st}(v, T)|$ is an arc. Let $C_{1}$ and $D_{1}$ denote two such components. By Lemma 3 of [10] $\bar{C}_{1}$ contains an element $s_{1}$ of $T_{2}$ that is free in $D$. If $\mid$ st $\left(v_{1}, T\right) \mid$ is not free in $D$ for some 0 -simplex $v_{1}$ of $s_{1}$ in Int $(D)$, then select $C_{2}$ a component of $D-\left|s t\left(v_{1}, T\right)\right|$ not intersecting $D_{1}$. Now there exists $s_{2} \in T_{2}$ so that $s_{2} \subset \bar{C}_{2}$ and is free in $D$. This process must stop eventually to produce a cell $t_{1}$ with the desired property. Analogously, we find $t_{2} \subset \bar{D}_{1}$.

If, at the beginning of the argument all of the conclusion follows but the last part of (2), then Lemma 3 of [10] and the argument above may be applied to the component of $D-\left|\mathrm{st}\left(v_{1}, T\right)\right|$.

LEMMA 1. Suppose that $T$ is a finite rectilinear triangulation of the boundary of the bounded, connected, simply connected open subset $D$ of the plane. Then, there is a triangulation $S$ of $\bar{D}$ such that (1) $T \subset S$, and (2) $n\left(S_{2}\right) \leqq a+2 b-2$, where $b$ is the number of elements $w$ of $T_{1}$ such that $\operatorname{seg} w \subset \operatorname{Int}(\bar{D})$ and $a=n\left(T_{1}\right)-b$.

Proof. The proof is by induction on $n=n\left(T_{1}\right)$, the case $n=3$ being obvious. Suppose for all cases $i=3, \ldots, n-1$.

Let $v$ belong to $T_{0}$ and the convex hull of $\bar{D}$ and let $U$ denote the union of all intervals $v p$ such that $\operatorname{seg} v p \subset D$ and $p \in \mathrm{Bd}(D)$.

If for no such $v p$ is $p \in T_{0}$ then a subset of $T_{1}$ may be expressed as $v p_{1}, \ldots, v p_{m}$ where $M=\bigcup_{i=1}^{m-1}\left(\operatorname{seg} p_{i} p_{i+1} \cup\right.$ Int $\left.v p_{i} p_{i+1}\right) \subset U$ and $v p_{1} \cup v p_{m} \subset \operatorname{Bd}(\bar{D})$. The desired triangulation is generated by the 2-cells $v p_{i} p_{i+1}(i=1, \ldots, m-1)$ together with those cells obtained by applying the induction hypothesis to $D-\bar{M}$ and the obvious triangulation of its boundary.

If for some $v p$ above $p \in T_{0}$, then $D-v p$ is the union of two mutually separated sets $D_{1}$ and $D_{2}$. The induction hypothesis is then applied to $D_{1}$ and $D_{2}$ and the obvious triangulations of their boundaries. 
LEMma 2. Suppose that $J_{0}, J_{1}, \ldots, J_{n}(n \geqq 0)$ is a collection of mutually exclusive polyhedral subsets of the plane $E^{2}$ such that (1) there is a bounded, connected, simply connected, open subset $D_{0}$ of $E^{2}$ such that $J_{0}=\mathrm{Bd}\left(D_{0}\right)$, (2) if $1 \leqq p \leqq n$, then $J_{p}$ is a simple closed curve which is a subset of $D_{0}$, and (3) if $0 \leqq p \leqq n$, then $J_{p}$ has a triangulation $T^{p}$. Then, $\bar{D}_{0}$ has a triangulation $T$ such that (1) $\bigcup_{p=0}^{n} T^{p} \subset T$ and (2) $n\left(T_{2}\right) \leqq a+2\left(b+\sum_{p=1}^{n} n\left(T_{1}^{p}\right)\right)-2$, where $b$ is the number of 1-simplexes $w$ of $T^{0}$ such that seg $w \subset$ Int $\left(\bar{D}_{0}\right)$ and $a=n\left(T_{1}^{0}\right)-b$.

Proof. The proof is by induction on $n$, the case $n=0$ reducing to Lemma 1 . Suppose true for all cases $j=0,1, \ldots, n-1$.

The boundary of the convex hull of $\bigcup_{p=1}^{n} J_{p}$ may be denoted by $A_{1} A_{2} \cdots A_{s} A_{1}$ where each of $A_{1} A_{2}, \ldots, A_{s} A_{1}$ is an interval and $\left\{A_{1}, \ldots, A_{s}\right\} \subset \bigcup_{p=1}^{n} T_{0}^{p}$.

Consider a typical $A_{p}$. Since $\bigcup_{q=1}^{n} J_{q} \subset D_{0}$, there is a point of $J_{0}$ in the interior of the angle vertical to angle $A_{p-1} A_{p} A_{p+1}$, which then implies there is an interval $X A_{p}$ where $X \in T_{0}^{0}$ and $\operatorname{seg} X A_{p} \subset D_{0}-\bigcup_{p=1}^{n} J_{p}$.

Suppose, for simplicity of notation, that $A_{p} \in J_{1}$ and that $J_{2}, \ldots, J_{t}$ are the ones of $J_{0}, \ldots, J_{n}$ that are subsets of Int $J_{1}$, and that $J_{t+1}, \ldots, J_{n}$ are subsets of $D_{0}-$ $\mathrm{Cl}\left(\right.$ Int $\left.J_{1}\right)$. It is easily verified that $\left\{J_{0} \cup X A_{p} \cup J_{1}, J_{t+1}, \ldots, J_{n}\right\}$ and $\left\{J_{1}, \ldots, J_{t}\right\}$ together with triangulations $\left\{T^{0} \cup T^{1} \cup\left\{X A_{p}\right\}, T^{t+1}, \ldots, T^{n}\right\}$ and $\left\{T^{1}, \ldots, T^{t}\right\}$, respectively, satisfy the induction hypothesis. The triangulations of $\mathrm{Cl}\left(D_{0}-\operatorname{Int} J_{1}\right)$ and $\mathrm{Cl}\left(\operatorname{Int} J_{1}\right)$ thus obtained may be combined to give the desired collections.

LEMMA 3. Let $K$ denote a polygonal knot in regular position in $E^{3}$ such that the $x y$ projection $\pi K$, of $K$, has exactly $n$ crossing points. Then, there is a polygonal knot $L$ in regular position such that (1) there is a p.l. homeomorphism $h$ from $E^{3}$ onto $E^{3}$ such that $h(K)=L$, and $h$ takes lines parallel to the z-axis onto lines of that type, and (2) $\pi L$ is the union of $\leqq 2^{n+2}-2$ intervals, no two of which have more than a common endpoint common, and $L$ itself is the union of $\leqq 2^{n+2}-2$ intervals, where in this case no endpoint of one of the intervals is projected into a crossing point of $\pi L$.

Proof. In case $n=1$, it is straightforward to show that $L$ may be constructed so that it is the union of 4 intervals and $\pi L$ is the union of 6 intervals. Now suppose the theorem holds for all cases $j=1, \ldots, n-1$.

First we note that an oriented knot $M$ whose projection has exactly $n$ double points has the property [14, Theorem 19] that if $a_{i} b_{i}(i=1,2)$ are intervals which lie in $M$ and whose projections cross at $e$, an interior point of each, and which contain no other double point of $\pi M$, then (assuming that the orientation of $M$ goes from $a_{i}$ to $\left.b_{i}(i=1,2)\right)$ a new knot $M^{\prime}$, whose projection has exactly $n-1$ crossings, can be made from $M$ by picking $c_{i}, d_{i} \in a_{i} b_{i}$ in the order $a_{i} c_{i} e_{i} d_{i} b_{i}$ (where $\left.\pi e_{i}=e\right)$ and letting $M^{\prime}=\left(M-\left(c_{1} d_{1} \cup c_{2} d_{2}\right)\right) \cup c_{1} c_{2} \cup d_{1} d_{2}$.

On the other hand, suppose we take a knot projection $P$ with orientation $O_{1}$ induced by an orientation of the knot, and consider a complementary domain $D$ of $P$. $D$ has a boundary $a_{1} a_{2} \cup a_{2} a_{3} \cup \cdots \cup a_{i} a_{1}$ (where each $a_{p} a_{p+1}$ denotes a straight 
line interval and where $\mathrm{Bd}(D)=\mathrm{Bd}(\bar{D}))$ (see [14, Theorem 19]) such that we may choose in $D$ close to $\mathrm{Bd}(D)$ a simple closed curve $J=b_{1} b_{2} \cup \cdots \cup b_{i} b_{1}$ so that each $b_{p}$ is close to $a_{p}$ and each $b_{p} b_{p+1}$ is a straight line interval. Let $J$ be given an orientation and let that orientation induce a circular orientation $\mathrm{O}_{2}$ on $\mathrm{Bd}(D)$.

Now suppose that $a_{r} a_{r+1}$ and $a_{s} a_{s+1}$ are such that $O_{1}$ and $O_{2}$ agree on $a_{r} a_{r+1}$ if and only if they agree on $a_{s} a_{s+1}$. Noting that $r$ may be $s$, we choose points $b, c \in \operatorname{seg} a_{r} a_{r+1}$ and $d, e \in \operatorname{seg} a_{s} a_{s+1}$ in the respective orders $a_{r} b c a_{r+1}, a_{s} d e a_{s+1}$; and if $r=s$ we also let $c$ precede $d$. Let $b x e$ and $c x d$ denote polygonal arcs which (1) lie in $D$ except for their endpoints and (2) contain intervals which cross at $x$, but where bxe $\cap c x d=\{x\}$. The set $P^{\prime}=(P-(b c \cup d e)) \cup b x e \cup c x d$ is a knot projection having exactly one more crossing point than $P$. Using [10], [14] it can be shown that every knot projection of $n$ crossings can be made from one of $n-1$ crossings by the above construction. We now return to the original problem.

By the former construction construct a knot $K^{\prime}$ from $K$ where $\pi K^{\prime}$ has exactly $n-1$ crossing points. By the induction hypothesis there is a homeomorphism $f$ and a knot $L^{\prime}$ such that conditions (1) and (2) hold relative to $K^{\prime}, L^{\prime}, f^{\prime}$ and $n-1$. Thus, $\pi L^{\prime}$ is the union of $\leqq 2^{n+1}-2$ intervals, no two of which have more than a common endpoint common. The projection $P$ of the required knot $L$ is now constructed from $\pi L^{\prime}$ from the latter construction described above.

In case $r=s$, then $P$ is constructed from $\pi L^{\prime}$ by adding a single loop to a side of a complementary domain. Such a $P$ can be formed with no more than $6+2^{n+1}-2$ and thus with no more than $2^{n+2}-2$ intervals.

In case $r \neq s$, we observe that $i$ is no more than $2^{n+1}-2$. Also the arcs bxe and $c x d$ can be required to "travel" around $\mathrm{Bd}(D)$ in the "shortest" direction. We find that $P$ can be formed with no more than $2+2\left(2^{n+1}-2\right)=2^{n+2}-2$ intervals.

The required knot $L$ is then built above $P$ so as to have the correct over and under crossings. The required map $h$ is built by first finding a p.l. homeomorphism $h^{\prime}$ from $E^{2}$ onto $E^{2}$ taking $\pi K$ onto $\pi L$, and then $h$ is defined so as to take $K$ onto $L$ in such a way that $h^{\prime}(\pi x)=\pi h(x)$ for $x \in E^{3}$.

The proof of Lemma 3 may now be altered slightly to yield

LEMMA 3'. As in Lemma 3 except that the conclusion is (a) weakened by replacing $2^{n+2}-2$ by $2^{n+2}$, and (b) strengthened by requiring that $\pi L$ be the union of intervals which are parallel to either the $x$-axis or the $y$-axis.

Lemma 4. Suppose that $M_{i}(i=1,2,3)$ is a polyhedral $p$-cell $(p=2$ or 3$)$ in $E^{p}$ which has a rectilinear triangulation $T^{i}$. Let $f_{i}: M_{i} \rightarrow M_{i+1}(i=1,2)$ be a p.l. homeomorphism which is affine on each $s \in T^{i}$. Then, there is a subdivision $T$ of $T^{1}$ such that (1) $f_{2} f_{1}$ is affine on each $s \in T$, and (2) $n\left(T_{p}\right) \leqq\left(3-p+2^{3 p-4}\right) n\left(T_{p}^{1}\right) n\left(T_{p}^{2}\right)$.

Proof for $p=3$. For $t_{1} \in T_{3}^{1}$ and $t_{2} \in T_{3}^{2}$ consider the convex set $t=t_{2} \cap f_{1}\left(t_{1}\right)$, if it has a nonvoid interior. Each flat face $w$ of $t$ is a subset of a face of $f_{1}\left(t_{1}\right)$ or $t_{2}$ and is a 2 -cell bounded by $q$ sides for $q \in\{3,4,5,6\}$. For each such $w$ if $q>3$ we 
use Lemma 1 to triangulate $w$ into $q-2$ triangular disks. Now each such $t$ as above is triangulated radially from some $P \in \operatorname{Int}(t)$ using sets of the form $K=P w^{\prime}$ where $w^{\prime}$ is either a $w$ with 3 sides or a 2-cell of the triangulation of $w$ if $q>3$. Since each $t$ has at most 8 such faces $w$, we find that the subdivision of $T^{1}$ whose 3 simplexes are sets of the form $f_{1}^{-1}(K)$ satisfies the conclusion of the lemma.

Lemma 5. In $E^{p}$ ( $p=2$ or 3) suppose $M$ is a polyhedral $p$-cell with triangulation $T$ and that $C \neq M$ is in $T_{p}$ and free in $M$. Then, there is a subdivision $T^{\prime}$ of $T$ such that (1) $n\left(T_{p}^{\prime}\right) \leqq(11 p-18) n\left(T_{p}\right)$ and (2) there is a simplicial isotopy $h: M \times[0,1] \rightarrow M$ such that (a) $h_{t}$ is affine on each $s \in T^{\prime}$ for each $t \in[0,1]$, (b) $h_{t}(x)=x$ if $t=0$ or $x \in \mathrm{Bd}(M)-C$, and (c) $h_{1}(M)=\mathrm{Cl}(M-C)$.

Proof for $p=3$. We follow Sanderson in Theorem 5 of [10]. Let $n$ be the number of 2-cell faces of $C$ lying in Bd $(M)$. There is exactly one $n-1$ simplex $P$ of $C$ not lying in $C \cap \mathrm{Bd}(M)$, and a unique $3-n$ simplex $Q$ which is not in $\mathrm{Cl}(M-C)$, and is opposite $P$ in the sense that it is the only $3-n$ simplex of $C$ not containing $P$.

Let $p_{1}$ be the centroid of $P$ and $q_{1}$ the centroid of $Q$. Let $p_{2}$ be a point on the extension of $p_{1} q_{1}$ through $p_{1}$, close enough to $p_{1}$ so that $K_{p}=p_{2}(C \cap \operatorname{Bd}(M))$ $\subset$ Int $\mid$ st $(P, T) \mid \cup(C \cap \mathrm{Bd}(M))$. Let $q_{2}$ be a point on the extension of $p_{1} q_{1}$ through $q_{1}$, close enough to $q_{1}$ such that $K_{q}=q_{2}(C \cap \mathrm{Bd}(M)) \subset\left(E^{3}-M\right) \cup$ $(C \cap \mathrm{Bd}(M))$. Still following Sanderson, we define $\bar{h}\left(q_{1}, t\right)$ to be the point of $p_{1} q_{1}$ a distance of $t d\left(p_{1} q_{1}\right)$ from $q_{1}$. For each $t$ we let $\bar{h}$ be fixed outside $K_{p} \cup K_{q}$ and let $\bar{h}$ map every interval joining a point $x$ of $M \cap \mathrm{Bd}\left(K_{p}^{\prime} \cup K_{q}\right)$ to $q_{1}$ linearly onto the interval joining $x$ to $\bar{h}\left(q_{1}, t\right)$. Define $h=\left.\bar{h}\right|_{M}$.

Let $C$ be denoted by abcd. We show how to obtain the triangulation $T^{1}$ only in the most difficult case, namely $Q=a b c$ and $p_{1}=P=d$. We subdivide $C$ into solid tetrahedra $a b p_{1} q_{1}, a c p_{1} q_{1}$ and $b c p_{1} q_{1}$, and do not subdivide any element of $T$ which does not have $p_{1}$ as a vertex. If $D \in T_{3}$ has exactly a 2-simplex common with $C$, we subdivide $D$ into two tetrahedra, $D \cap K_{p}$ and $\mathrm{Cl}\left(D-K_{p}\right)$.

Now suppose $D \in T_{3}$ where $D \cap C \in T_{0} \cup T_{1}$ and $p_{1} \in D$. Then, there is a minimal triangulation $T^{D}$ of $\mathrm{Bd}(D) \cup\left(D \cap \mathrm{Bd}\left(K_{p}\right)\right)$ such that (1) if $f$ is a 2-cell face of $D$ having no interior point common with $K_{p}$ then $f \in T^{D}$, (2) every 2-simplex in $T^{D}$ lying in $\operatorname{Bd}(D) \cap K_{p}$ has $p_{1}$ as a vertex. $D$ is then subdivided into sets of the form $x w$ where (a) $x=p_{1}$ and $w \in T_{2}^{D}$ lying in $K_{p}$ but where $p_{1} \notin w$, or (b) $x$ is a fixed point of Int $\left(D-K_{p}\right)$ and $w \in T_{2}^{D}$ and $w \subset \operatorname{Bd}\left(\operatorname{Int}\left(D-K_{p}\right)\right)$. (There is a fixed $x$ in Int $\left(D-K_{p}\right)$ so that if $y, z$ are two points of $\mathrm{Bd}\left(\operatorname{Int}\left(D-K_{p}\right)\right)$ then $x y$ and $x z$ are different rays from $x$.) Remembering that in forming the $T_{2}^{D}$ 's a face $f$ must be subdivided the same way from both sides, $T^{1}$ is generated by the 3 -simplexes described above.

In the worst possible case (which occurs when $p_{2} \in \operatorname{Int}(D)$ ) $D$ is subdivided into $\leqq 25$ tetrahedra. In all other cases $D$ is subdivided into no more than 15 tetrahedra and if $D \cap C$ is a 2-cell, $D$ is subdivided into 2 tetrahedra. Thus, the number 15 in the conclusion of the lemma. 
LEMMA 6. In $E^{p}(p=2$ or 3$)$ suppose that $M$ is a polyhedral $p$-cell with triangulation $T$ and that $C \neq M$ is a p-simplex of $T$ that is free in $M$. Let

$$
S=\{t: t \in T \text { and } t \subset \mathrm{Cl}(M-C)\} .
$$

Then, there is a subdivision $S^{\prime}$ of $S$ such that (1) $n\left(S_{p}^{\prime}\right) \leqq(11 p-18) n\left(T_{p}\right)$ and (2) there is a simplicial isotopy $\mathrm{g}: \mathrm{Cl}(M-C) \times[0,1] \rightarrow M$ such that (a) $g_{t}$ is affine on each $s \in S^{\prime}$ for each $t \in[0,1]$, (b) $g_{t}(x)=x$ if $t=0$ or $x \in \operatorname{Bd}(M)-C$, and (c) $g_{1}(\mathrm{Cl}(M-C))=M$.

Proof for $p=3$. Let $h$ and $T^{\prime}$ be as in Lemma 5 and define $S^{\prime}=\left\{t: t \in T^{\prime}\right.$ and $t \subset \mathrm{Cl}(M-C)\}$. Define $g$ by $g(x, t)=h(x, 1-t)$ for $x \in \mathrm{Cl}(M-C)$ and $t \in[0,1]$.

Definition. Given two points $x$ and $y$ of the interior of the compact convex set $C$, we define the $S$-isotopy $h$ determined by $(x, y, C)$ so that (1) $h$ is first defined by $h_{t}(x)=(1-t) x+t y$ for $t \in[0,1],(2)$ if $P \in \mathrm{Bd}(C)$ then $P x$ is mapped linearly onto $P h_{t}(x)$ for $t \in[0,1]$ and (3) $h_{t}(P)=P$ for any element of domain $h$ outside $C$.

LEMMA 7. Suppose $P$ is a compact polyhedral 3-manifold with boundary in $E^{3}$ and $S$ is a rectilinear triangulation of $\mathrm{Bd}(P)$. Then, there is a triangulation $T$ of $P$ such that $n\left(T_{3}\right) \leqq(j-3) j 2^{j}$, where $j=n\left(S_{2}\right)$.

Proof. Let $W$ denote the set of all planes which contain an $s \in S_{2}$ and let $g$ denote a component of $P-(U W)$. Then $\bar{g}$ is a convex 3-cell having $\leqq j$ flat faces, each of which is bounded by a simple closed curve which is the union of $\leqq j-1$ straight line intervals. Each flat face is then triangulated (using Lemma 1) into no more than $(j-1)-2$ 2-cells, and then $\bar{g}$ is radially subdivided from some $x \in g$ into no more than $j(j-3)$ solid tetrahedra. Since there are at most $2^{j} g$ 's the bound above is evident.

Lemma 8. Suppose (1) $P, S$ and $j$ are as in Lemma 7, (2) the triangular disk $a b c \subset$ Int $(P),(3) K$ is a subset of $P$ so that $\bar{K}$ is a polyhedron and $\bar{K} \cap a b c \subset\{a, c\}$, and (4) $y$ is the midpoint of ac. Then, there is a triangulation $W$ of $P$ and a simplicial isotopy $g: P \times[0,1] \rightarrow P$ such that (1) $g_{t}(x)=x$ if $t=0$ or $x \in\{a, b\} \cup K \cup \operatorname{Bd}(P)$, (2) $g_{1}(b)=y\left(\right.$ or $\left.g_{1}(y)=b\right)$, (3) $n\left(W_{3}\right) \leqq(j+8)(j+5) 2^{j+8}+12+8\left(3+(3 j) 2^{3 j}\right)$, and (4) $g_{t}$ is affine on each $w \in W$ for each $t \in[0,1]$.

Proof. The method of proof is a combination of ideas in Lemma 2 of [1] and Lemma 5. Let $a x c$ and $a z c$ be triangular disks such that $x, z$ lie on line $y b$ in the order $x y b z$, and $a x c \cup a c z \subset($ Int $(P)-\bar{K}) \cup\{a, c\}$. Let $q_{1} p_{1} y p_{2} q_{2}$ be a short straight line interval which pierces disk axc $\cup$ acz at $y$, and has the property that the union $Q$ of the tetrahedra $q_{i} a c x, q_{i} a c z(i=1,2)$ is a subset of (Int $\left.(P)-\bar{K}\right) \cup\{a, c\}$.

The map $g$ is defined as in Lemma 5 on $y$ or $b$, depending on which is to be moved to which, and then extended linearly on intervals joining $y$ (or $b$ ) to $\mathrm{Bd}(R)$, where $R$ is the union of $\operatorname{axcp}_{i}, \operatorname{aczp}_{i}(i=1,2)$. Elsewhere, let $g(p, t)=p$. The elements of $W_{3}$ are now described. 
(1) Let $p_{i} a x y, p_{i} c x y, p_{i} a y b, p_{i} c y b, p_{i} a b z, p_{i} c b z(i=1,2)$ be elements of $W_{3}$.

(2) The methods of Lemma 7 are used to triangulate $\mathrm{Cl}(P-Q)$, where the 2-simplexes in the triangulation of the boundary either belong to $S$ or are a face of one of $q_{i} a c x, q_{i} a c z(i=1,2)$.

(3) The tetrahedra $p_{i} q_{i} a x, p_{i} q_{i} x c, p_{i} q_{i} a z, p_{i} q_{i} c z(i=1,2)$ are triangulated the same way. For, consider $p_{1} q_{1} a x$. In the triangulation of $\mathrm{Cl}(P-Q)$, the face $q_{1} a x$ has been subdivided into $\leqq(3 j) 2^{j}$ triangular disks, so $p_{1} q_{1} a x$ is radially subdivided from some interior point $t$ into $\leqq 3+(3 j) 2^{j}$ tetrahedra, including $t p_{1} a x, t p_{1} q_{1} x$ and $t p_{1} q_{1} a$.

DEFINITION. Let the bound given in part (3) of the conclusion of Lemma 8 be denoted by $G(j)$.

\section{The proofs of theorems.}

THEOREM 1. Suppose $\Delta$ is a solid tetrahedron in $E^{3}, D_{i}(i=1,2)$ is a polyhedral disk in Int $(\Delta)$ with triangulation $T^{i}$, and $h: \Delta \rightarrow \Delta$ is an onto p.l. homeomorphism such that (a) $h(x)=x$ if $x \in \operatorname{Bd}(\Delta)$, and (b) $h\left(D_{1}\right)=D_{2}$. Then, there is a simplicial isotopy $\phi: \Delta \times[0,1] \rightarrow \Delta$ such that $(1) \phi_{t}(x)=x$ if $t=0$ or $x \in \mathrm{Bd}(\Delta),(2) \phi_{1}\left(D_{1}\right)=D_{2}$, and (3) there is a triangulation $T$ of $\Delta$ such that (a) $\phi_{t}$ is linear on each $s \in T$ for each $t \in[0,1]$ and (b) $n\left(T_{3}\right) \leqq(32 G(4))^{8+2 N_{1}+2 N_{2}}$, where $N_{i}=28\left(n\left(T_{2}^{i}\right)+1\right)\left(16 n\left(T_{2}^{i}\right)+12\right)$, $i=1,2$.

Proof. Let $w_{1}, \ldots, w_{n_{1}}$ denote a shelling order [10] of $D_{1}$, where each $w_{p} \in T_{2}^{1}$. Let $w_{1}=a c b$. We consider two cases.

Case 1. $a b c \cap \mathrm{Cl}\left(D_{1}-a b c\right)=b c$. Let $d b c \in T_{2}^{1}-\left\{w_{1}\right\}$. (i) If $a, b, c$ and $d$ are coplanar, let $g: \Delta \times[0,1] \rightarrow \Delta$ denote an $S$-isotopy such that (1) $g(x, t)=x$ if $t=0$ or $x \in \mathrm{Bd}(\Delta) \cup \mathrm{Cl}\left(D_{1}-(a b c \cup b c d)\right) \cup\{b, c\}$, (2) $g_{1}\left(D_{1}\right)=\mathrm{Cl}\left(D_{1}-a b c\right)$, and (3) there is a triangulation $T^{g}$ of $\Delta$ so that (a) $g_{t}$ is affine on each $s \in T^{g}$ for each $t \in[0,1]$, and (b) $n\left(T_{3}^{g}\right) \leqq G(4)$. (ii) Suppose $a, b, c$ and $d$ are not coplanar. Let $e=\frac{1}{2}(b+c)$ and let $x \in$ line de such that (a) $x \in \operatorname{Int}(\Delta)-D_{1}$ and (b) $b c x a-a c b$ $\subset$ Int $(\Delta)-D_{1}$. We use the "composition" of two $S$-isotopies $g, g^{\prime}$ as above, where $g$ moves $a c b$ onto $a c x$ and is fixed on $D_{1} \cup \mathrm{Bd}(\Delta)$ and where $g^{\prime}$ moves $x b c$ into $b c d$ analogous to $g$ in (i) above. We also assume the bound $G(4)$ for each of $g, g^{\prime}$.

Case 2. $a b c \cap \mathrm{Cl}\left(D_{1}-a b c\right)=a c \cup b c$. Utilizing the proof of Lemma 2 of [1], let $J$ denote the simple closed curve $\mathrm{Bd}\left|\mathrm{st}\left(c, T_{1}\right)\right|$ and let $J^{\prime}$ denote the simple closed curve which is the projection of $J$ on $\Delta$ from $c$. Since $J$ is the union of no more than $n_{1}=n\left(T_{2}^{1}\right)$ straight line intervals, then $J^{\prime}$ is the union of no more than $3 n_{1}$ intervals each of whose endpoints are (a) the projection on $\Delta$ of a point $x$ where $c x \in T_{1}^{1}$ or (b) on a 1-simplex of $\Delta$. The simple closed curve $W=$ (plane $a b c$ ) $\cap \Delta$ is the union of no more than 4 intervals having their endpoints on the 1-skeleton of $\Delta$. By Lemma 1 there is a triangulation $R$ of $\mathrm{Bd}(\Delta)$ such that (1) $W \cup J^{\prime} \subset\left|R_{1}\right|$, (2) $n\left(R_{1}\right) \leqq 28\left(n_{1}+1\right)$ and (3) $n\left(R_{2}\right) \leqq 16 n_{1}+12$. 
Let $a^{\prime}\left(b^{\prime}\right)$ denote the projection of $a(b)$ from $c$ on $\Delta$, let $A_{1}=a^{\prime} x_{1}^{\prime} b^{\prime}$ denote the $\operatorname{arc~} \mathrm{Cl}\left(J^{\prime}-a^{\prime} b^{\prime}\right)$, and let $a^{\prime} x_{1} b^{\prime}=B_{1}=\mathrm{Cl}\left(W-a^{\prime} b^{\prime}\right)$, where we assume $a^{\prime} b^{\prime}$ is the projection on $\Delta$ from $c$ of interval $a b$. There is a sequence of $\operatorname{arcs} A_{1}, \ldots, A_{N_{1}}=B_{1}$ such that (1) $A_{p} \subset\left|R_{1}\right|-\operatorname{seg} a^{\prime} b^{\prime}, p=1, \ldots, N_{1}$, (2) if $1 \leqq p<N_{1}$, then there is an element $s_{p}$ of $R_{2}$ so that $A_{p}-s_{p}=A_{p+1}-s_{p}$ and $\mathrm{Bd}\left(s_{p}\right) \subset A_{p} \cup A_{p+1}$, and (3) $N_{1} \leqq 28\left(n_{1}+1\right)\left(16 n_{1}+12\right)$.

Now suppose, for example, that $s_{1}=x y z$ where $A_{1} \cap s_{1}=x y \cup y z$ and $A_{2} \cap s_{1}$ $=x z$. Let $s=\frac{1}{2}(x+z)$ and let $x^{\prime}, y^{\prime}, z^{\prime}, r^{\prime}, s^{\prime}, t^{\prime}$ denote points so that (1) $x^{\prime}, y^{\prime}, z^{\prime}$ are elements of $J$ whose projections from $c$ are $x, y, z$, respectively, (2) $s^{\prime}$ is on $x^{\prime} z^{\prime}$ and the projection of $s^{\prime}$ from $c$ on $\Delta$ is $s$, (3) $r^{\prime}$ and $t^{\prime}$ lie on line $y^{\prime} s^{\prime}$ in the order $t^{\prime} s^{\prime} y^{\prime} r^{\prime}$, and (4) the union $K$ of the tetrahedra $c x^{\prime} r^{\prime} z^{\prime}, c x^{\prime} z^{\prime} t^{\prime}, s x^{\prime} r^{\prime} z^{\prime}$, and $s x^{\prime} z^{\prime} t^{\prime}$ is a subset of $\{s\} \cup \operatorname{Int}(\Delta)$ and intersects $\mid$ st $\left(a, T_{1}\right) \mid$ in the union of two triangular disks $c x^{\prime} y^{\prime}$ and $c y^{\prime} z^{\prime}$. Let $g^{1}$ be the $S$-isotopy determined by $\left(y^{\prime}, s^{\prime}, K\right)$.

Analogously, $S$-isotopies $g^{2}, \ldots, g^{N}$ are defined so that their "composition" together with $g^{0}$ moves $\mid$ st $\left(c, T_{1}\right) \mid$ to a planar set while keeping $a b c$ fixed. Then an $S$-isotopy $h$ is defined so that (1) $h_{t}(x)=x$ if $t=0$ or $x \in g_{1}^{N} \cdots g_{1}^{1}\left[\mathrm{Cl}\left(D_{1}-\mid\right.\right.$ st $\left.\left.\left(c, T_{1}\right) \mid\right)\right]$ $\cup \operatorname{Bd}(\Delta)$, (2) $h_{1} g_{1}^{N} \cdots g_{1}^{1}\left(D_{1}\right)=\mathrm{Cl}\left(g_{1}^{N} \cdots g_{1}^{1}\left(D_{1}-a b c\right)\right)$. Finally, we let $v^{1}=\left(g^{1}\right)^{-1}$ $\cdots\left(g^{N}\right)^{-1} h g^{N} \cdots g^{1}$, which concludes Case 2 .

Using Case 1 or 2 as needed for $w_{2}, \ldots, w_{n_{1}-1}$ we construct simplicial isotopies $V^{2}, \ldots, V^{n_{1}-1}$ such that (a) $V_{1}^{n_{1}-1} \cdots V_{1}^{1}\left(D_{1}\right)=w_{n_{1}}$ and (2) each $V^{p}$ is a composition of no more than $2 N_{1}+1 S$-isotopies, where the bound $G(4)$ applies to each factor.

Let $u_{1}, \ldots, u_{n_{2}}\left(n_{2}=n\left(T_{2}^{2}\right)\right)$ denote a shelling order for $D_{2}$, where each $w_{p} \in T_{2}^{2}$. As above there is a sequence of simplicial isotopies $m^{1}, \ldots, m^{u_{2}-1}$ whose composition takes $D_{2}$ onto $u_{n_{2}}$ and each $m^{p}$ is a composition of no more than $2 N_{2}+1$ $S$-isotopies. Also, $w_{n_{1}}$ can be moved onto $u_{n_{2}}$ by an isotopy $q$ which is the composition of no more than $6 S$-isotopies, where the bound $G(4)$ applies to each factor.

The required map $\phi$ is defined by $\left(m^{1}\right)^{-1} \cdots\left(m^{n_{2}-1}\right)^{-1} q V^{n_{1}-1} \cdots V^{1}$ and is the composition of no more than $6+2 N_{1}+1+2 N_{2}+1 S$-isotopies. Applications of Lemmas 4 and 8 finish the proof.

THEOREM 2. Suppose that (1) $K$ and $L$ are polygonal knots in regular position in $E^{3}$ such that the $x y$ projections $\pi K$ and $\pi L$ lie on different sides of some line in the $x y$ plane, and have $M$ and $N$ crossing points, respectively, and (2) $G_{K}$ and $G_{L}$ are collections of straight line intervals such that (a) $\pi K=\bigcup G_{K}$ and $\pi L=\bigcup G_{L}$, (b) no two intervals of $G_{K} \cup G_{L}$ have more than an endpoint common, and (c) $n\left(G_{K}\right) \leqq 2^{M+2}$ -2 and $n\left(G_{L}\right) \leqq 2^{N+2}-2$. Then, there is a solid tetrahedron $A B C D$ with triangulation $T$ such that (1) $K \cup L \subset\left|T_{1}\right| \cap$ Int $(A B C D),(2) n\left(T_{3}\right) \leqq 64\left(2^{M+2}+2^{N+2}\right)-8(M+N)$ +96 , and (3) if $C$ is a 3-cell which is the union of a nondegenerate subcollection $G$ of $T_{3}$, then there exist two elements of $G$ that are free in $C$.

Proof. Suppose each point in $K \cup L$ has a positive $z$ coordinate. Let $A, B, C, F$ denote points in the $x y$ plane such that (1) line $C F$ separates $\pi K$ from $\pi L$, (2) 
$F \in \operatorname{seg} A B$, and (3) $\pi K \subset$ Int $(A C F)$ and $\pi L \subset$ Int $(B C F)$. Let $D$ denote a point so high above the barycenter of $A B C$ that the projection $P(K \cup L)$ from $D$ through $K \cup L$ into the $x y$ plane has the properties (1) $P(K) \subset \operatorname{Int}(A C F)$ and $P(L) \subset$ Int $(B C F)$, and (2) there is a p.l. homeomorphism $h$ from disk $A B C$ onto $A B C$ such that (a) $h(x)=x$ if $x \in C F \cup \mathrm{Bd}(A B C)$, (b) $h(\pi K)=P(K)$ and $h(\pi L)=P(L)$ and (c) if $g \in G_{K} \cup G_{L}$ then $h(g)$ is a straight line interval. There exist points $H$ and $E$ and intervals $g_{1} \in G_{k}, g_{2} \in G_{L}$ such that $H$ is an endpoint of $h\left(g_{1}\right), E$ is an endpoint of $h\left(g_{2}\right)$, and intervals $C H, C E, H F$ and $E F$ have the property that $P(K)-H$ is a subset of the interior of the simple closed curve $A F H C A$ and $P(L)-E \subset$ Int $(F B C E F)$. Note also that a knot projection of $j$ crossings has exactly $j+2$ complementary domains, and that no arc lying in the projection and not containing a crossing point is a subset of the boundaries of more than two complementary domains. Let

$$
S=\left\{h(g): g \in G_{K} \cup G_{L}\right\} \cup\{A F, F B, B C, C A, H C, H F, F E, E C, C F\} .
$$

Consider a typical complementary domain $U$ of $A B C-|S|$. Bd $(U)$ may be expressed as the union of straight line intervals $a_{1} a_{2}, \ldots, a_{n} a_{1}$ where (1) each $a_{p} a_{p+1} \in S$ and (2) there is a polygonal simple closed curve $b_{1} b_{2} \cdots b_{1} b_{n}$ in $U$ so that (a) each $b_{p}$ is close to $a_{p}$, and (b) each $b_{p} b_{p+1}$ is a straight line interval. Each quadrilateral disk $a_{p} b_{p} a_{p+1} b_{p+1}$ is triangulated as follows: Choose $c_{p}, e_{p}, f_{p} \in$ $\operatorname{seg} a_{p} a_{p+1}$ in the order $a_{p} c_{p} e_{p} f_{p} a_{p+1}$, choose $d_{p} \in \operatorname{seg} b_{p} b_{p+1}$, and subdivide the quadrilateral disk into $a_{p} c_{p} b_{p}, c_{p} b_{p} d_{p}, c_{p} e_{p} d_{p}, e_{p} f_{p} d_{p}, f_{p} d_{p} b_{p+1}$ and $f_{p} a_{p+1} b_{p+1}$.

In a given quadrilateral we have used at most six 2-simplexes. Also, remembering the addition of the $d_{p}$ 's, the disk bounded by $b_{1} b_{2} \cdots b_{n} b_{1}$ may be triangulated with no more than $2 n-22$-simplexes. Thus, a typical $\bar{U}$ with $\mathrm{Bd}(U)=a_{1} a_{2} \cdots a_{n} a_{1}$ as above is triangulated into $\leqq 6 n+2 n-22$-simplexes. Then, if $W$ is the triangulation of $A B C$ with these 2-simplexes, then $n\left(W_{2}\right) \leqq 16\left(2^{M+2}+2^{N+2}\right)-2(M+N)+24$. Note that if an $a_{p} a_{p+1}$ in Int $(A B C)$ is triangulated one way from one side it must be triangulated the same way from the other side.

We now proceed to construct the collection $T$. Given $w \in W_{2}$, if $D w$ does not intersect $K \cup L$, let $D w \in T_{3}$. If $D w$ intersects $K \cup L$ there are three cases to consider. Let $w=a b c$.

Case 1. $(K \cup L) \cap D w$ is a single point $d$ in the interior of a 1-simplex, say $a D$. We subdivide $D w$ into solid tetrahedra $D d b c$ and $d a b c$.

Case 2. $(K \cup L) \cap D w$ is a straight line interval de where $d \in \operatorname{seg} a D, e \in \operatorname{seg} D c$. Subdivide $D w$ into $b d e D, b d e c$, and $a b c d$, provided $c$ corresponds to the role of $c_{p}$ or $f_{p}$ above.

Case 3. $(K \cup L) \cap D w$ is the union of a straight line interval de and a point $g$, where $g, d \in \operatorname{seg} a D$ and $e \in \operatorname{seg} D c$.

(a) If $g \in \operatorname{seg} D d$, subdivide $D w$ into $b g e D, b d e g, b d e c$, and $a b c d$.

(b) If $g \in \operatorname{seg} d a$, subdivide $D w$ into $b d e D$, decb, $a b c g$, and $d c g b$. 
We now let $T$ denote the triangulation of $A B C D$ whose 3-simplexes are of the form $D w$ where $D w \subset A B C D-(K \cup L)$ or a subset of a $D w$ as given in Cases $1,2,3$. Since no $D w$ is subdivided into more than 4 tetrahedra, then $n\left(T_{3}\right) \leqq 4 n\left(W_{2}\right)$, the stated bound.

Now let $C=\bigcup G$ where $G$ is a nondegenerate subset of $T_{3}$ and $C$ is a 3-cell, and let $R=\left\{w \mid w \in W_{2}\right.$ and $D w$ contains an element of $\left.G\right\}$. Let $V=|R|$, and for each $w \in R$ let $G_{w}=\{g: g \in G$ and $g \subset D w\}$. If $V_{1}$ denotes the set of all 1-simplexes $t$ of elements of $R$ where $t \subset \operatorname{Int}(V)$ and $t$ does not contain the $P$ projection (from $D$ ) of a point of Int $(C)$, then we find that $V-\left|V_{1}\right|$ is connected and simply connected. So, if we imagine that $V$ is "split open" along the simplexes in $V_{1}$, then the new $R$ (say $R_{s}$ ) yields a cellular subdivision of the new $V$ (say $V_{s}$ ). By Lemma 0 either $R_{s}$ is degenerate or there are two 2-cells $w, w^{\prime}$ of $R_{s}$ that are free in $V_{s}$ and have the following additional property:

$\left(\mathrm{P}_{1}\right)$ If $v$ is a 0 -simplex of $w$ (or $\left.w^{\prime}\right)$ and $v \in \operatorname{Int}\left(V_{s}\right)$, then $\left|\mathrm{st}\left(v, R_{s}\right)\right|$ is free in $V$, and if $v, v^{\prime}$ are 0 -simplexes of $w, w^{\prime}$, respectively, that lie in $\operatorname{Int}\left(V_{s}\right)$ and $W_{0} \cap \operatorname{Int}\left(V_{s}\right)$ is nondegenerate, then $v \neq v^{\prime}$.

If $R_{s}$ is degenerate, then it is easy to see that the various cases yield two elements of $F$, the set of all elements of $G$ free in $C$. We therefore suppose that $w, w^{\prime}$ are two elements of $R_{s}$ free in $V$, where property $\left(\mathrm{P}_{1}\right)$ holds, and that we are also proceeding by induction on the number of elements in $G$, supposing the desired result to hold for all such $G$ 's with fewer elements. The basic idea of the proof is to show outright there are two elements of $F$ or to show there exists $f \in F$ having a certain property relative to $w$, i.e. either $f \subset w$, or if $v \neq v^{\prime}$ as in $\left(\mathrm{P}_{1}\right)$, then $f$ has a 0 or 1 -simplex on $v D$ and would not have the same property relative to $v^{\prime} D$.

If $w$ has no vertex in Int $\left(V_{s}\right)$, it is straightforward to show in all the cases that (1) if $D w$ contains at least two elements of $G$, then $D w$ contains two elements of $F$, and (2) if $D w$ contains only one element $g$ of $G$ then $g \in F$, and the induction hypothesis may be applied to $\mathrm{Cl}(C-g)$ to produce another element of $F$. We thus now assume that $w$ and $w^{\prime}$ have 0 -simplexes $v$ and $v^{\prime}$, respectively, in $\operatorname{Int}\left(V_{s}\right)$, and that property $\left(\mathrm{P}_{1}\right)$ holds.

Now suppose that $g \in G$ and $g$ has two 2-cell faces $f_{1}, f_{2}$ such that $f_{1} \cup f_{2} \subset \operatorname{Bd}(C)$. (a) If $g \cap \mathrm{Bd}(C)=f_{1} \cup f_{2}$ or $f_{1} \cup f_{2} \cup f_{3}$, where $f_{3}$ is a third 2-cell face of $g$, then $g \in F$. (b) The other possible case is that $g \cap \mathrm{Bd}(C)=f_{1} \cup f_{2} \cup s$, where $s$ is a 1 -simplex of $g$, and $g \notin F$. There is then a third face $f_{3}$ of $g$ such that $C-f_{3}$ is the union of two mutually separated sets $C_{1}, C_{2}$, and the induction hypothesis may be applied to $\bar{C}_{i}$ to produce a free cell $g_{i}(i=1,2)$ such that $g_{i}$ does not have $f_{3}$ as a face unless $g_{i}=\bar{C}_{i}$. Then $g_{1}, g_{2} \in F$. Hereafter, we shall assume

$\left(\mathrm{S}_{1}\right)$ In the above circumstances case (a) always holds.

Remembering that $V$ has been split along certain 1-simplexes to form $V_{s}$, we show how to handle the argument in the more difficult cases. We suppose that $w=a b c$ has only one 1-simplex lying in $\mathrm{Bd}\left(V_{s}\right)$, and where $D w$ is subdivided as in Case 3(a) above. Using the notation of 3(a) let $A_{1}, \ldots, A_{10}$ denote the 2-simplexes $b g D, b g d, b d a, D g e, g d e, d e c, a d c, b e D, b e c$, and $a b c$, respectively. 
Case (F1). $a c=a b c \cap \mathrm{Bd}\left(V_{s}\right)$. In this case $\mid$ st $(b d, T) \mid \subset C$ so bge $D \in G_{w}$. Since every element of $G_{w}$ has a 2-simplex on $a c D$, then some element of $G_{w}$ has two 2-simplexes lying in $\mathrm{Bd}(C)$. By assumption $\left(\mathrm{S}_{1}\right), G_{w}$ contains an element of $F$.

Case (F2). $a b=a b c \cap \mathrm{Bd}\left(V_{s}\right)$. Either (a) $|\mathrm{st}(D e, T)| \subset C$ or (b) $|\mathrm{st}(e c, T)| \subset C$. In either case the only way for $\left(\mathrm{S}_{1}\right)$ to fail to yield an $f \in F \cap G_{w}$ is for

$$
G_{w}=\{b g d D, \text { bged }, \text { bdec }\}
$$

and for $\bigcup_{i \in J}$ Int $\left(A_{i}\right) \subset \operatorname{Int}(C)$, where $J=\{4,5,6,8,9\}$. There exist triangles $a b c$, $b c r, c f r, c f u, c u t$, and atc in $W_{2}$ such that in forming $T$ (1) Dabc is as above, (2) $D b c r$ is subdivided into bcre and berD, (3) Drcf is subdivided into Dres, rcfe, and rfes, (4) Ducf is subdivided into Desu, ucfe, and uefs, (5) Dcut is subdivided into teuD and tuce and (6) Dtac is subdivided into tgeD, dtge, tdec, and tacd.

Suppose (a) holds. Then bge D, berD, Ders, Desu, Detu, and Degt are also in G. Also, by the conditions on $\bigcup_{i \in J} A_{i}$ above, becr, tged and tdec are in $G$.

At this stage we consider the two cases:

(i) $c$ is the only 0 -simplex of $W_{0}$ in Int $\left(V_{s}\right)$ (and thus $\bigcup\{a b, a t, t u, u f, f r, b r\}$ $=\mathrm{Bd}\left(V_{s}\right)$ ).

(ii) There are at least two elements of $W_{0}$ in Int $\left(V_{s}\right)$.

Suppose (i) holds. If recf $\notin G$ then becr and one of resf and Desr are in $F$. So suppose $r e c f \in G$. If $u c f e \notin G$, then $r c f e$ and one of uesf and ues $D$ are in $F$. Suppose $u c f e \in G$. If uesf $\notin G$, then $u c f e$ and ues $D$ are in $F$, so suppose uesf $\in G$. If tuce $\notin G$, then $u e f c$ and teuD are in $F$, so suppose tuce $\in G$. At this stage we have $\mid$ st $(e, T) \mid$ $\subset C$, so bge $D$ is in $F$ and is a subset of $D w$.

Now suppose (ii) holds. If $r e c f \in G$, then $c e b r \in F$, where $c e b r \cap c D=c e$, so suppose $r e c f \in G$. If $e s f r \notin G$, then $e c f r \in F$, so suppose $e s f r \in G$. If $e c f u \notin G$, then

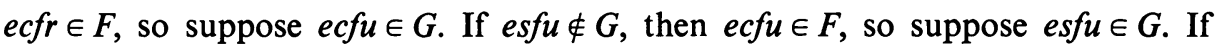
tuce $\notin G$, then $e c f u \in F$, so suppose tuce $\in G$. As above we have $\mid$ st $(e, T) \mid \subset C$, so bge $D \in F$.

We now omit Case (F2(b)) and proceed to

Case (F3). $a b c \cap \mathrm{Bd}\left(V_{s}\right)=b c$. In this case either (a) $\mid$ st $(g D, T) \mid \subset C$, or (b) $\mid$ st $(g d, T) \mid \subset C$, or (c) $\mid$ st $(a d, T) \mid \subset C$.

Assuming, as explained above, that $a \in \operatorname{Int}\left(V_{s}\right)$, we label the 3-simplexes of $T$ that contain a point of $a D$. Dabc is subdivided as above, and in each of the seven lines below the left-hand tetrahedron is the union of the four on the right.

$a b p D ; \quad a b p d, p d g b, y g b p, y g D b$

qapD; apqd, qpdg, qpgy, qgyD

arqD; arqd, rdqs, $s d g q, s D g q$

tarD; tard, rsdt, sdgt, sgDt

tavD; tavd, tgdv, tguv, tguD

vamD; vamd,vdmg, uvgm, ugm D

macD; macd, mdec, mdeg, mge $D$ 
In Case (F3) we show how to handle only case (b) above, all critical ideas of the others being represented here. Recalling assumption $\left(S_{1}\right)$ above, we find that $D w$ contains an element of $G$ obviously in $F$ or else (i) $G_{w}=\{$ bged, bge $D\}$ or (ii) $G_{w}=\left\{t \in T_{3}: t \subset D_{w}\right\}$ or (iii) $G_{w}=\{b g e d, b d e c, a b c d\}$. In each of these three cases, if $t \in T_{3}$ and $t \cap t^{\prime}=s$ for $t^{\prime} \in G_{w}$ and $s$ a 2-simplex subset of Dab $\cup$ Dac, then $t$ is assumed to be in $G$. Otherwise, there is an element of $F$ in $D w$.

Suppose case (i) of case (b) now holds. Suppose also $\{a\}=W_{0} \cap \operatorname{Int}\left(V_{s}\right)$. If $y g p b \notin G$, then $y g D b$ and one of $p g d b$ and $a b p d$ are in $F$, so suppose $y g p b \in G$. Also, since at least two elements of $G$ are in st $(g D, T)$ we assume $\mid$ st $(g D, T) \mid \subset C$; otherwise we can find two elements of $F$. Now, we also assume that every element $T$ of $T_{3}$ lying directly below $t^{\prime} \in$ st $(g D, T) \cap G$ and having a 2-simplex common with $t^{\prime}$ is in $G$. For suppose, for example, that $g y p q \notin G$. Then, $g y q D \in F$ and an element of $\{q d g p\} \cup$ st $(a d, T)$ is in $F$. We now have the case $\mid$ st $(g, T) \mid \subset C$, so $g e b D \in F$.

Still supposing that $\{a\}=W_{0} \cap \operatorname{Int}\left(V_{s}\right)$, we handle cases (ii) and (iii) above. Since there are at least two 3-simplexes of st $(a d, T)$ in $G$ we suppose they all are, for otherwise we obtain two elements of $F$. If $r s d q \notin G$ then raqd and one of $r d a t$ and $r d s t$ are in $F$. So suppose that $r s d q \in G$ and, analogously, that $r s d t \in G$. We now have $\mid$ st $(d, T) \mid \subset C$, so abcd $\in F$.

We now handle case (b)(i) in the case that $W_{0} \cap \operatorname{Int}\left(V_{s}\right)$ is nondegenerate. Since $a b c d \notin G$, we may as well assume no element of st $(a d, T)$ is in $G$, for if one is, then one is in $F$ and has a 1 -simplex common with $a D$. If $p g y b \notin G$, then $p d b g \in F$ and has a 1-simplex common with $a D$. So suppose $p g y b \in G$, and, analogously, that $q g y p, \operatorname{tguv}, \operatorname{uvgm} \in G$. If $q g y D \notin G$, then $q \in \operatorname{Bd}\left(V_{s}\right)$ so (1) $\bigcup\{b c, b p, q p\} \subset \mathrm{Bd}\left(V_{s}\right)$ or (2) $\bigcup\{r q, r t, t v, v m, m c, b c\} \subset \mathrm{Bd}\left(V_{s}\right)$. But case (1) implies $g y D b \in F$ and (2) implies one of $s d g q, r s d q$, and $s g D q \in F$. Thus we assume $q g y D \in G$ and, likewise, that $u g m D, \operatorname{tg} u D \in G$. If $\operatorname{sg} D q \notin G$ then $q \in \operatorname{Bd}\left(V_{s}\right)$, and as above we find an element of $F$. So suppose $s g D q$ and likewise $s g D t \in G$. We now have the case $\mid$ st $(g, T) \mid \subset C$, so bgde $\in F$.

Consider now cases (b)(ii),(iii) where $W_{0} \cap \operatorname{Int}\left(V_{s}\right)$ is nondegenerate. Since $a b c d \in G$, we may as well assume $\mid$ st $(a d, T) \mid \subset C$. If $r s d q \notin G$, then $\operatorname{raqd} \in F$. So suppose that $r s d q$ and, likewise, $r s d t \in G$. We now have $\mid$ st $(d, T) \mid \subset C$, so $a b c d \in F$. This completes the proof for Case (F3(b)). Since the arguments given are typical of, and as difficult as, the other cases in Theorem 2, this completes the proof given for Theorem 2.

\section{REFERENCES}

1. R. H. Bing, An alternative proof that 3-manifolds can be triangulated, Ann. of Math. (2) 69 (1959), 37-65. MR 20 \#7269.

2. R. H. Crowell and R. H. Fox, Introduction to knot theory, Ginn, Boston, Mass., 1963. MR 26 \#4348.

3. C. F. Gauss, Werke (8), Teubner, Leipzig, (1900), pp. 272, 282-286. 
4. M. Greendlinger, Dehn's algorithm for the word problem, Comm. Pure Appl. Math. 13 (1960), 67-83. MR 23 \#A1693.

5. - On Dehn's algorithms for the conjugacy and word problems, with applications, Comm. Pure Appl. Math. 13 (1960), 641-677. MR 23 \#A2327.

6. - On the word problem and the conjugacy problem, Izv. Akad. Nauk SSSR Ser. Mat. 29 (1965), 245-268. (Russian) MR 30 \#4820.

7. J. V. Sz.-Nagy, Über ein topologisches Problem von Gauss, Math. Z. 26 (1927), 579-592.

8. L. P. Neuwirth, Knot groups, Ann. of Math. Studies, no. 56, Princeton Univ. Press, Princeton, N. J., 1965. MR 31 \#734.

9. D. E. Penney, An algorithm for establishing isomorphism between tame prime knots in $E^{3}$, Dissertation, Tulane University, New Orleans, La., 1965.

10. D. E. Sanderson, Isotopy in 3-manifolds. I. Isotopic deformations of 2-cells and 3-cells, Proc. Amer. Math. Soc. 8 (1957), 919-922. MR 19, 760.

11. — , Isotopy in 3-manifolds. II. Fitting homeomorphisms by isotopy, Duke. Math. J. 26 (1959), 387-396. MR 21 \#5956.

12. P. G. Tait, On knots, Scientific Paper I, Cambridge Univ. Press, London, 1898.

13. L. B. Treybig, Prime mappings, Trans. Amer. Math. Soc. 130 (1968), 248-253. MR 36 \#879.

14. - A characterization of the double point structure of the projection of a polygonal knot in regular position, Trans. Amer. Math. Soc. 130 (1968), 223-247. MR 36 \#878.

15. - An approach to the polygonal knot problem using projections and isotopies, Trans. Amer. Math. Soc. 158 (1971), 409-421.

TULANE UNIVERSITY,

New OrLeans, Louisiana 70118 